\title{
The Difference in Calcium Levels in Aspergillus nidulans Grown on Glucose or Pectin
}

\author{
Janice Aparecida Rafael, Suraia Said ${ }^{*}$ \\ Departamento de Ciências Farmacêuticas, Faculdade de Ciências Farmacêuticas de Ribeirão Preto, \\ Universidade de São Paulo, São Paulo, Brazil \\ Email: "susaid@usp.br
}

Received March 16, 2012; revised April 5, 2012; accepted April 15, 2012

\begin{abstract}
Understanding the growth regulatory mechanisms in filamentous fungi is very important for the production of medicines for antifungal therapies. It is well established that $\mathrm{Ca}^{2+}$ gradient is essential for hyphal growth and that one mechanism responsible for the $\mathrm{Ca}^{2+}$ cellular concentration starts with the hydrolysis of phosphatidylinositol 4,5bisphosphate $\left(\mathrm{PIP}_{2}\right)$ by receptor-regulated forms of phosphoinositide-specific phospholipase C (PI-PLC). In the present study the levels of calcium in Aspergillus nidulans wild type (A26) and plcA-deficient mutant (AP27) growing in a carbon source readily assimilated, as glucose or pectin a non-readily assimilated carbon source was investigated. Intracellular calcium levels in A26 were higher in the presence of glucose than in pectin, but lower in AP27 independently of the carbon source and in AP27 the vesicular calcium distribution occurred mainly at the apex of the hyphae. Delay in nuclear division was also observed if A26 and AP27 were grown in pectin presence when compared with growth in glucose. For the first time, it is demonstrated that the levels of intracellular $\mathrm{Ca}^{2+}$ were higher when A. nidulans was growing in glucose than in a non readily assimilated carbon source as pectin. Further, it also showed that the plcA gene, although not essential, may be responsible for high-molecular weight carbon source recongnation, for the intracellular $\mathrm{Ca}^{2+}$ levels maintenance and consequently by the nuclear division in A. nidulans.
\end{abstract}

Keywords: Aspergillus nidulans; $\mathrm{Ca}^{2+}$ Signaling; Phospholipase C

\section{Introduction}

Calcium ions act as second messengers in the signal transduction pathways in eukaryotic organisms from fungi to humans. The key features of the $\mathrm{Ca}^{2+}$ machinery in fungi are conserved as multicellular eukaryotes and underlie the diverse fungal physiological processes [1]. A tip-high $\mathrm{Ca}^{2+}$ gradient is an essential requirement for polarized hyphal growth [2] and $\mathrm{Ca}^{2+}$ channel blockers or ionophores affect the hyphal growth, morphogenesis, and branching [3]. It is known that one of the mechanisms responsible for regulating the $\mathrm{Ca}^{2+}$ cellular concentration starts with the hydrolysis of phosphatidylinositol 4,5bisphosphate $\left(\mathrm{PIP}_{2}\right)$ by receptor-regulated forms of phosphoinositide-specific phospholipase C (PI-PLC) generating two products, myo-inositol 1.4.5-triphosphate $\left(\mathrm{IP}_{3}\right)$ and di-0-acylglycerol (DAG), which are the second messengers in the control of many cell functions [4-6]. IP3 has a key role in calcium regulation as it activates the channels in the membrane-bound calcium storage vesicles, liberating the $\mathrm{Ca}^{2+}$ ions into the cytosol, which in its

\footnotetext{
"Corresponding author.
}

turn activates the calmodulin dependent enzymes.

Very little is known about the mechanisms, which coordinate morphogenesis, growth and nuclear division in fungi, mostly when they are growing in different carbon sources. Our previous results showed that two phospholipase C (E.3.1.4.3) (PLC) inhibitors delayed the first nuclear division in the $A$. nidulans cultures growing in the glucose presence, but stimulated it in a medium with pectin [7]. Thus, it was suggested that PLC may modulate differently the cellular signal related with the first nuclear division depending on glucose or pectin to be the carbon source present. On the other hand plcA appears to be linked to high-molecular weight carbon source sensing since wild type strain decreased nuclear division rates when cultivated in media with polypectate, whereas AP27 did not show slow nuclear duplication rates when grown on this carbon source [8]. As IP3, a product of a reaction catalyzed by PLC is also responsible for intracellular calcium regulation, the present study describes the relations of intracellular calcium levels and two carbon sources in a mutant transformed with plcA gene rupture growing in the presence of readily (glucose) or non-readily (pectin) assimilated carbon source. The re- 
sults show for the first time the importance of carbon source and PLC in the maintenance of intracellular calcium levels and consequently in the first stages of germination in A. nidulans.

\section{Materials and Methods}

\subsection{Strains, Chemicals, Media and Growth Conditions}

A. nidulans, FGSC A26 (biA1, veA1) designated A26 here and the mutant transformed with plcA gene rupture referred as AP27 [8], which was originally FGSC A851 (pabaA1 yA2; argB::trpC_B;veA1 trpC801) were used throughout the present study. The strains were maintained by weekly transfers onto complete medium (CM) slants as described by Vanzela and Said [9] and incubated at $30^{\circ} \mathrm{C}$ for 7 days before the experiments. Cove's MM (pH 6.0) contained $0.5 \%(\mathrm{w} / \mathrm{v})$ glucose or pectin as the sole carbon source and was supplemented with 0.02 $\mu \mathrm{g} / \mathrm{ml}$ biotin for A26 or para-aminobenzoic acid (PABA) for AP27. Chlortetracycline (CTC), 4,6-diamidino-2-phenylindole (DAPI) and dimethylsulfoxide (DMSO) were purchased from Sigma Aldrich Co. (St. Louis, Mo, USA). Fluo-3-acetoxymethyl-ester (Fluo-3/AM) was from Molecular Probes, Eugene, Oregon, USA. The other chemicals, media, and reagents were either from Sigma or of an analytical grade bought elsewhere.

\subsection{Analysis of Nuclear Division and Calcium Distribuition}

To determine the spatial distribution of the $\mathrm{Ca}^{2+}$-containing organelles, the mycelium pre-grown for $30 \mathrm{~h}$ at $30^{\circ} \mathrm{C}$ were fixed as described by Kawano and Said [10] and $100 \mu \mathrm{M}$ of CTC were directly applied onto the hyphae [11]. To analyse the nuclear division liquid MM supplemented with glucose or pectin $0.5 \%(\mathrm{w} / \mathrm{v})$ was inoculated with $10^{6}$ conidia $/ \mathrm{mL}$ and poured on Petri dishes containing sterile glass cover slides and incubated at $30^{\circ} \mathrm{C}$. After $8 \mathrm{~h}$ of incubation, the cover slides with adhered conidia and germlings were removed with tweezers and processed for microscopy. Nuclei were stained with DAPI as described by Kawano and Said [10]. Images of organellar calcium fluorescence and the nuclei were viewed under a fluorescence microscope (Axioskope) and images captured by AxioCam HRc, both from Zeiss. The results of at least three independent experiments are reported as mean $\pm \mathrm{SD}$.

\subsection{Intracellular $\mathrm{Ca}^{2+}$ Detection by Flow Cytometry}

Intracellular $\mathrm{Ca}^{2+}$ was determined with the $\mathrm{Ca}^{2+}$-sensitive fluorescence dye Fluo-3/AM, dissolved in DMSO and stored in aliquots protected from light at $4^{\circ} \mathrm{C}$. Conidia suspensions $\left(10^{6}\right)$ from A26 and AP27 strains pre-incubated for $8 \mathrm{~h}$ in liquid MM containing $1 \mathrm{mM}$ of ethylene glycol tetra acetic acid (EGTA), to chelate extracellular calcium, were centrifuged at $472 \times \mathrm{g}$ for 5 minutes and resuspended in $1 \mathrm{~mL}$ water. Conidia and germlings were transferred to individual tubes $(0.5 \mathrm{~mL})$, loaded with Fluo-3/AM $(25 \mu \mathrm{M})$ and incubated for $35 \mathrm{~min}$ at $25^{\circ} \mathrm{C}$. During incubation Pluronic F-127, a nonionic poliol, was added to a final concentration of $1 \%$ to facilitate dye penetration. DMSO and Pluronic F-127 were also added to the controls. Analyses were performed on a FACSCanto (BD San Jose-USA) and the FACSDiva software was used for the acquisition of 10,000 events collected for each sample on FACSArray. The Fluo-3 fluorescence was detected using a 530/30 nm filter. Two groups of cells were detected by flow cytometer; P8 cells with Fluo-3 fluorescence signal negative and P9 cells with Fluo-3 fluorescence signal positive.

\subsection{Statistical Analysis}

Experiments were done in duplicate or triplicate and independently repeated. The bars in the figures indicate the mean values \pm standard deviation (SD). Data were statistically analyzed by one-way ANOVA followed by Bonferroni's $t$ test and considered significantly different when $\mathrm{P}<0.05$ was obtained.

\section{Results and Discussion}

\subsection{Spatial Distribution of $\mathrm{Ca}^{2+}$}

CTC fluorescence, indicating the $\mathrm{Ca}^{2+}$ containing vesicles, was observed after $30 \mathrm{~h}$ of incubation in glucose or pectin. CTC fluorescence was detected in subapical hyphal region and mainly in apex of A26 strain grown in glucose (Figure 1(A)), while in pectin the fluorescence was in all hyphal extension (Figure 1(C)). Thus, it was suggested that the different calcium distributions dependent on the fungus growth were in glucose or pectin. On the other hand, hyphae of the AP27 mutant showed CTC fluorescence only in the apex independently of the carbon source (Figures 1(B) and (D)). Alterations of $\mathrm{Ca}^{2+}$ spatial distribution was also detected using CTC in Neurospora crassa when the IP3-induced $\mathrm{Ca}^{2+}$ release was inhibited. In this condition, the tip-high $\mathrm{Ca}^{2+}$ fluorescence disappeared and was replaced by increased $\mathrm{Ca}^{2+}$ fluorescence behind the tip [12]. In some fungi, such as Candida albicans [13], Saccharomyces cerevisiae [14] and Neurospora crassa [15] $\mathrm{Ca}^{2+}$ may be released from vacuolar vesicles by IP3 activation. Herein, the obtained results demonstrated alterations in the $\mathrm{Ca}^{2+}$ spatial distribution in AP27 strain of $A$. nidulans probably due the low IP3 levels as a consequence of plcA gene rupture. 

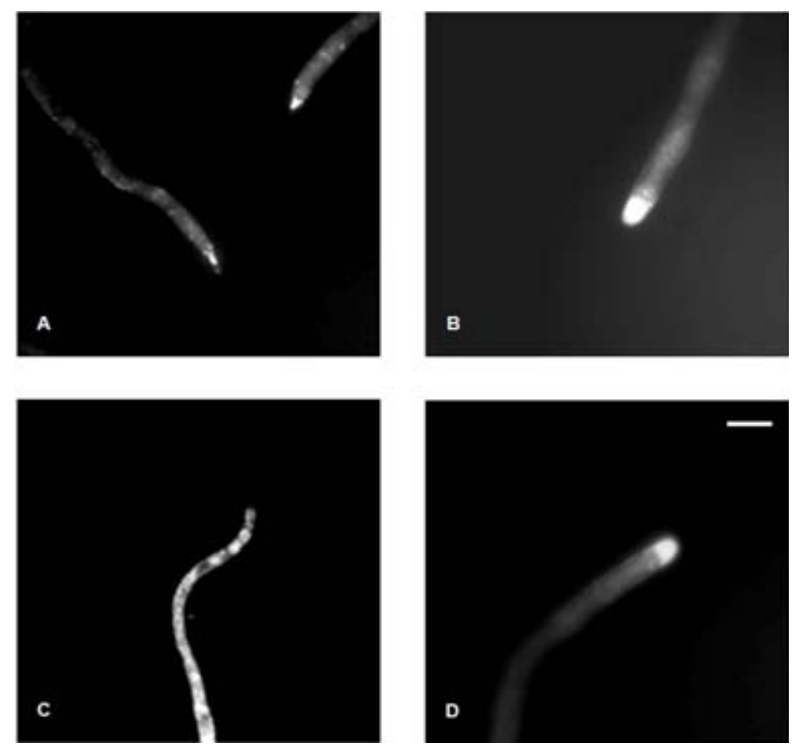

Figure 1. Analysis of vesicular $\mathrm{Ca}^{2+}$ in hyphae of $A$. nidulans A26 and AP27 strains. The hyphae A26 (A and C) and AP27 (B and D) strain were pre-grown in $(0.5 \%)$ glucose (A and $B)$ or pectin (C and D). CTC $(100 \mu M)$ was applied onto the mycelium for 15 minutes. Vesicular $\mathrm{Ca}^{2+}$ fluorescence was visualized under fluorescence microscopy. Bars $1 \mathrm{~cm}$.

\subsection{Intracellular Calcium Levels and Nuclear Division in Different Culture Conditions}

Fluo-3/AM was used for the first time in A. nidulans to detect intracellular calcium by flow cytometry. In conidia and germlings of A26 incubated in media supplemented with glucose or pectin, fluorescence was detected in approximately $22 \%$ and $4 \%$ cells, respectively, while in AP27 the percentages were approximately 11 and 3.5, respectively. Thus, it was demonstrated that A26 had two times more intracellular $\mathrm{Ca}^{2+}$ than AP27 when growing in glucose, but in pectin-supplemented media, the strains had similar values, which were about 2 to 5 times smaller than those detected in glucose presence (Figure 2).

No significant differences were detected on conidial germination when A26 or AP27 was being cultivated in pectin or glucose presence but the percentage of cells contained four nuclei was bigger in both cultures growing in glucose than those in pectin (Figure 3) suggesting a delay in nuclear division if A26 and AP27 mutant were grown in pectin. Muller et al. [16] reported influence of glucose concentration on nuclear mitosis, septation and tip extension rate in Aspergillus oryzae and A. niger. The authors observed that the average nuclei numbers in hyphae apical compartments were higher at increased growth rates, which also happened at the highest glucose concentration. It was also suggested that fungi may be capable of regulating the average number of nuclei to a specific growth rate. This was viewed as an adaptation which was advantageous to the organism as the necessary energy levels for nuclear division depended on the
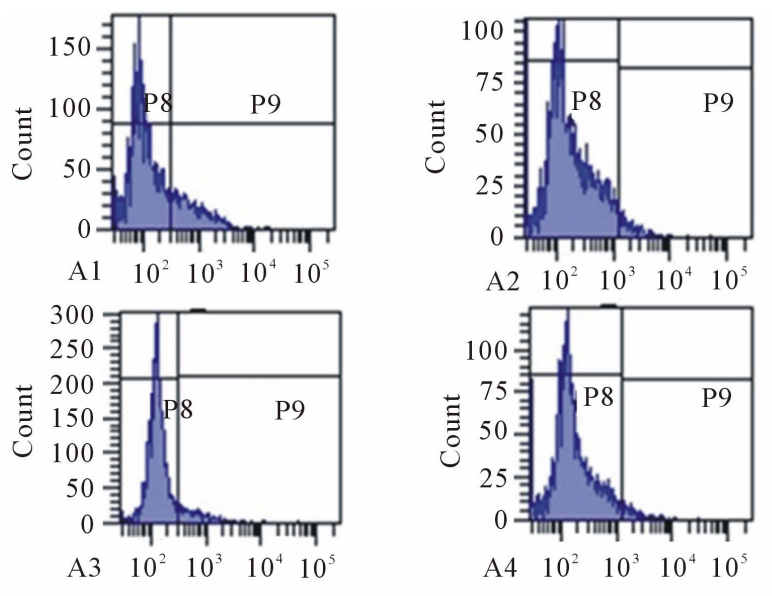

(A)

\begin{tabular}{ccc}
\hline Strain & Carbon Source $(0.5 \%)$ & Cytosolic $\mathrm{Ca}^{2+}(\%)$ \\
\hline \multirow{2}{*}{ A26 } & Glucose & 22.5 \\
& Pectin & 4.1 \\
AP27 & Glucose & 10.8 \\
\hline
\end{tabular}

(B)

Figure 2. Evaluation of intracellular $\mathrm{Ca}^{2+}$ in $\mathrm{A} 26$ and AP27 strains pre-grown in glucose or pectin for 8 hours. Hystogram showing Fluo-3/AM ( $25 \mu \mathrm{M})$ labelled conidia monitored by flow cytometry. (A) corresponding to cells stained by the probe; $x$-axis: intensity of fluorescence (log scale); y-axis: number of events. P8 cells with Fluo-3 fluorescence signal negative and P9 cells with Fluo-3fluorescence signal positive. (A1) A26 cultured in glucose; (A2) A26 cultured in pectin; (A3) AP27 cultured in glucose; (A4) AP27 cultured in pectin. The insert $B$ shows the percent distribution of $\mathrm{Ca}^{2+}$ in both strains.

number of nuclei present.

The nuclear division might be activated by plcA when glucose is available and a polymeric carbon source as pectin or polypectate would lead to an opposite response [8]. The release of calcium from the storage vesicles is regulated by IP3, one of the products generated by PLC reaction, whose enzyme in turn is product of the plcA expression what may suggeste an explaination to the low calcium levels detected when A26 or AP27 were growing in the presence of pectin and EGTA chelating agent (Figure 2). By the present work it is clear that in A. nidulans the intracellular $\mathrm{Ca}^{2+}$ levels depend on if the carbon source present in the culture is readily assimilated or not; $\mathrm{Ca}^{2+}$ in turn coordinates nuclear duplication and metabolic pathways dependent on $\mathrm{Ca}^{2+}$. The plcA gene appears to be directly involved in the recognition of highmolecular-weight carbon source [8]. In a possible regulatory mechanism the plcA gene would be activated in glucose presence, PLC would hydrolyze PIP2 generating IP3 and DAG what would keep the $\mathrm{Ca}^{2+}$ levels, but if a high-molecular-weight carbon source as pectin was in the 


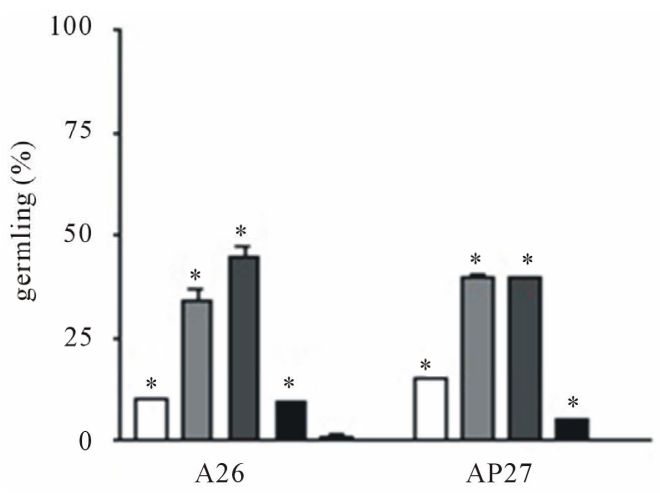

(A)

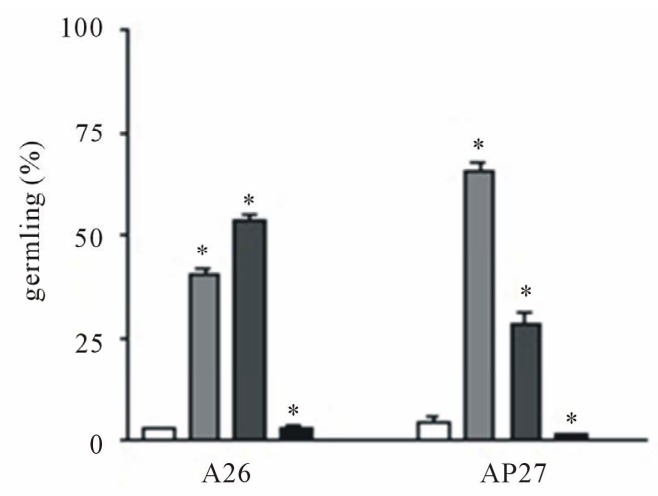

(B)

Figure 3. Effects of carbon source on conidial germination and nuclear division in A26 and AP27 strains. A26 and AP27 conidia were incubated in MM supplemented with (0.5\%) glucose (A) or pectin (B) for $8 \mathrm{~h}$. Results are represented by means \pm SD. ${ }^{*}$ Significantly different relative among A26 and AP27 strains $(P<0.05)$.

medium the plcA gene would be not activated conesquently the low $\mathrm{Ca}^{2+}$ levels would be not enough to realize the nuclear duplication and others activities at same rate observed in glucose. In AP27 mutant when PLC inhibitors spermine and $\mathrm{C} 48 / 80$ were used [7] and when sodium polypectate or pectin were the carbon source [8] delays were observed in nuclear duplication.

\section{Conclusion}

The results of the present study clearly showed that in $A$. nidulans, plcA gene and carbon source are not the unique but they are involved in maintenance of intracellular $\mathrm{Ca}^{2+}$ levels and consequently in other functions of cellular germination as on nuclear duplication. Other experiments have to be conducted using other different carbon sources and in other fungi to investigate if this is a general mechanism of regulation.

\section{Acknowledgements}

The present study is part of a thesis presented by J.A.R. to the Faculdade de Ciências Farmacêuticas de Ribeirão Preto, Universidade de São Paulo, in partial fulfillment of the requirements for the Doctor's degree. J.A.R. received a Doctoral Fellowship from Coordenação de Apoio de Pessoal de Nível Superior (CAPES). The authors are grateful to Dr. A. C. Tedesco for the Pluronic F-127 reagent, to F. R. Morais for assistance in flow cytometry analysis.

\section{REFERENCES}

[1] P. R. Kraus, C. B. Nichols and J. Heitman, "Calcium- and Calcineurin-Independent Roles for Calmodulin in Cryptococcus neoformans Morphogenesis and High-Temperature Growth," Eukariotic Cell, Vol. 4, No. 6, 2005, pp. 1079-1087. doi:10.1128/EC.4.6.1079-1087.2005

[2] O. Gavric, D. B. Santos and A. Griffiths, "Mutation and
Divergence of the Phospholipase C Gene in Neurospora crassa," Fungal Genetics and Biology, Vol. 44, No. 4, 2007, pp. 242-249. doi:10.1016/j.fgb.2006.09.010

[3] T. Sone and A. J. Griffiths, "The Frost Gene of Neurospora crassa Is a Homolog of Yeast cdcl and Affects Hyphal Branching via Manganese Homeostasis," Fungal Genetics and Biology, Vol. 28, No. 3, 1999, pp. 227-237. doi:10.1006/fgbi.1999.1169

[4] M. J. Berridge, "Inositol Triphosphate and Calcium Signaling," Nature, Vol. 361, No. 6410, 1993, pp. 315-325. doi: $10.1038 / 361315 \mathrm{a} 0$

[5] Y. Nishizuka, "Intracellular Signaling by Hydrolysis of Phospholipids and Activation of Protein Kinase C," Science, Vol. 258, No. 5082, 1992, pp. 607-614. doi:10.1126/science.1411571

[6] R. H. Michell, "Inositol Lipids in Cellular Signaling Mechanisms," Trends in Biochemical Sciences, Vol. 17, No. 8, 1992, pp. 274-276. doi:10.1016/0968-0004(92)90433-A

[7] M. A. S. C. Chellegatti, P. D. Yuvamoto and S. Said, "Role of Phospholipase C and Protein Kinase C in Aspergillus nidulans during Growth on Pectin or Glucose: Effects on Germination and Duplication Cycle," Folia Microbiologica, Vol. 55, No. 3, 2010, pp. 228-232. doi:10.1007/s12223-010-0033-6

[8] A. P. F. C. Vanzela, S, Said and R. A. Prade, "Phosphatidyl Inositol Phospholipase C Mediates Carbon Sensing and Vegetative Nuclear Duplication Rates in Aspergillus nidulans," Canadia Journal of Microbiology, Vol. 57, No. 7, 2011, pp. 611-616. doi:10.1139/w11-034

[9] A. P. F. C. Vanzela and S. Said, "Evidence for Carbon Source Regulated Protein Kinase A and Protein Kinase C Signaling in the Duplication Cycle, Polarization and Septum Formation in Aspergillus nidulans," Microbiologycal Research, Vol. 157, No. 3, 2002, pp. 239-247. doi:10.1078/0944-5013-00156

[10] C. Y. Kawano and S. Said, "Hyperbranching Induced by Cold-Shock or Snow-Flake Mutation in Neurospora crassa Is Prevented by Adition of Exogenous Calcium," Journal of Basic Microbiology, Vol. 45, No. 3, 2005, pp. 199- 
206. doi:10.1002/jobm. 200410496

[11] H. Prokisch, O. Yarden, M. Dieminger, M. Tropschug and I. B. Barthelmess, "Impairment of Calcineurin Function in Neurospora crassa Reveals Its Essential Role in Hyphal Growth, Morphology and Maintenance of the Apical $\mathrm{Ca}^{2+}$ Gradient," Molecular and General Genetics, Vol. 256, No. 2, 1997, pp. 104-114. doi:10.1007/s004380050551

[12] L. B. Silverman-Gavrila and R. R. Lew, "An IP3-Activated $\mathrm{Ca}^{2+}$ Channel Regulates Fungal Tip Growth," Journal of Cell Science, Vol. 115, No. 24, 2002, pp. 5013-5025. doi: $10.1242 /$ jes. 00180

[13] C. M. Calvert and D. Sanders, "Inositol TriphosphateDependent and Independent $\mathrm{Ca}^{2+}$ Mobilization Pathways at the Vacuolar Membrane of Candida albicans," The Journal of Biological Chemistry, Vol. 70, 1995, pp. 7272-
7280.

[14] P. J. M. Belde, J. H. Vossen, G. W. J. Borst-Pauwels and A. P. R. Theuvenet, "Inositol 1,4,5-Triphosphate Releases $\mathrm{Ca}^{2+}$ from Vacuolar Membrane Vesicles of Saccharomyces cerevisiae," FEBS Letters, Vol. 23, No. 1-2, 1993, pp. 113-118. doi:10.1016/0014-5793(93)81460-H

[15] G. Cornelius, G. Gebauer and D. Techel, "Inositol Triphosphate Induces Calcium Release from Neurospora crassa Vacuoles," Biochemical and Biophysical Research Communications, Vol. 162, No. 2, 1989, pp. 852-856. doi:10.1016/0006-291X(89)92388-7

[16] C. Muller, A. B. Spohr and J. Nielsen, "Role of Substrate Concentration in Mitosis and Hyphal Extension of Aspergillus", Biotechnology and Bioengineering, Vol. 67, No. 4, 2000, pp. 390-397. doi:10.1002/(SICI)1097-0290 